\title{
High Temperature Brillouin Scattering Study on Elastic Properties of Lithium Caesium Borate Glasses, Crystals and Melts
}

\author{
Shunsuke Aramomi ${ }^{1}$, Yu Matsuda ${ }^{1,2}$, and Seiji Kojima ${ }^{1 *}$ \\ ${ }^{1}$ Graduate School of Pure and Applied Sciences, University of Tsukuba, Tsukuba, Ibaraki 305-8573, Japan \\ ${ }^{2}$ Glass Research Center, Central Glass Co., Ltd., Matsusaka, Mie 515-0001, Japan
}

\begin{abstract}
The mixed alkali effect on elastic properties of lithium caesium borate glasses $0.25\left[x \mathrm{Li}_{2} \mathrm{O}-(1-x)\right.$ $\left.\mathrm{Cs}_{2} \mathrm{O}\right]-0.75 \mathrm{~B}_{2} \mathrm{O}_{3}$ was studied by Brillouin scattering. The observed mixed alkali effect on the negative deviation from linearity in the composition dependence of longitudinal modulus was discussed on the basis of the decrease of four-fold coordinated boron atoms and the increase of nonbridging oxygens. The temperature dependence of elastic properties was studied in the large temperature range from room temperature to $1500 \mathrm{~K}$ to clarify the differences between glasses, crystals and melts. The influence of annealing was clearly observed in LA sound velocity. Fragility was discussed using the temperature dependence of velocity.
\end{abstract}

Keywords: Alkali borate glass; Mixed alkaline effect; Elastic property; Brillouin scattering;

High temperature

*Corresponding Author E-mail: *kojima@ims.tsukuba.ac.jp (S. Kojima). 


\section{Introduction}

Generally many properties of oxide glasses shows the approximate linear changes with the composition. However, there is an important exception in alkali oxide glasses containing more than one alkali, and maximum of the electric registivity or the expansion coefficient appears [1]. Such mixed alkali effect (MAE) is known in oxide glasses, where two dissimilar alkali species coexist. Most of transport properties such as ionic conductivity and viscosity reveal remarkable deviation from the linearity when one alkali cation substitutes another at totally fixed concentration of alkalis. The reduction of diffusion in secnd alkali speicies was suggested, while its mechanism is unresolved problem [2]. The diffusion process was studied by local structure spectroscopies [3]. The theory of MAE was proposed to predict a negative entropy in mixing two dissimilar alkali species [4]. The concept of matrix-mediated coupling was proposed to discuss MAE by the assumption that coupling between movements of unlike cations in glass occurs in order to dissipate mechanical stresses created by small cations entering sites which are too large and large cations entering sites which are too small [5]. The reverse Monte Carlo caluculation on MAE showed that the two types of alkali ions are randomly mixed and have distinctly different conduction pathways of low dimensionality [6]. This implies that $\mathrm{A}$ ions tend to block the pathways for the $\mathrm{B}$ ions and vice versa, and this is the main reason for the MAE. The static physical properties such as density, elastic modulus also show not remarkable but noticeable deviaton from the liniar concentration dependence, while the mechanism of MAE on the static properties is not yet qulalified.

Nowadays, borates have become an important material in advanced materials, i.e., borate glasses [7] used for glassfiber and textile, or nonlinear optic crystals, such as $\mathrm{CsLiB}_{6} \mathrm{O}_{10}$ (CLBO) [8]. In binary borate glass system, the composition dependence of many different physical quantities exhibits an M-shape, or inversely a W-shape. The causes for these properties to show the M- or the $\mathrm{W}$-shapes have been analysed in terms of the three borate structural units, $\mathrm{BØ}_{3}, \mathrm{M}^{+} \mathrm{B}_{4}^{-}$, and $\mathrm{M}^{+} \mathrm{BO}_{2}{ }^{-}$, or in terms of glass network connectivity, where the symbol $\varnothing$ denotes bridging oxygen atoms that are shared between adjacent (super)structural units and the symbol, $\mathrm{O}$, denotes both bridging oxygen atoms that are situated completely within a superstructural unit, and negatively charged nonbridging oxygen atoms that form part of a (super)structural unit. .MAE on elastic and vibrational properties of lithium caesium borate glasses $0.28\left[x \mathrm{Li}_{2} \mathrm{O}-(1-x) \mathrm{Cs}_{2} \mathrm{O}\right]-0.72 \mathrm{~B}_{2} \mathrm{O}_{3}$ prepared by the melt-quench method was studied at room temperature by Brillouin scattering and Raman scattering spectroscopies [9]. MAE on elastic properties, density, and the low-energy excitation called a boson peak at room temperature were discussed, while the temperature dependence was not yet studied. Since the elastic properties of glass are fundamentally and technologically important, we extend the previous study at room temperature into the temperature dependence of elastic properties of glasses over the large temperature range including not only glassy but also crystalline and liquid states. Since, Brillouin scattering is the noncontact non-destructive method to determine the elastic properties, and it is very effective in comparison with the standard ultrasonic technique such as pulse-echo, resonance and pulsed laser ultrasonics, especially for the measurements at high temperatures. The study of elastic properties at high temperatures 
were studied by Brillouin scattering in alkali borate and alkali germinate germinate glasses [10,11]. In this study, we investigated the elastic properties of MAE of lithium caesium borate glasses in equilibrium liquid, supercooled liquid, glass, and crystalline states by Brillouin scattering spectroscopy.

\section{Experimental}

A series of lithium caesium borate glasses $y\left[x \mathrm{Li}_{2} \mathrm{O}-(1-x) \mathrm{Cs}_{2} \mathrm{O}\right]-(100-y) \mathrm{B}_{2} \mathrm{O}_{3}(y=0.25 ; x=0,0.1,0.25$, $0.5,0.75,0.9,1)$ were prepared with high homogeneity to investigate the inherent nature of the ternary glasses. The starting materials were analytical-reagent-grade $\mathrm{LiOH} \cdot \mathrm{H}_{2} \mathrm{O}, \mathrm{CsOH}$ and $\mathrm{H}_{3} \mathrm{BO}_{3}$. To achieve the high homogeneity of the samples, they were first reacted in an aqueous solution [11]. Then the mixed solution was dried at $140{ }^{\circ} \mathrm{C}$ until the water was completely evaporated. The dried powder was transferred to a platinum crucible, and heated in an electrical furnace at $1000{ }^{\circ} \mathrm{C}$ for $2 \mathrm{~h}$. The melt was then quenched by pressing between two aluminum plates. The quenched samples were annealed at a temperature range $380-490{ }^{\circ} \mathrm{C}$ depending on glass composition for $1 \mathrm{~h}$ and cooled at a rate of $1 \mathrm{~K} / \mathrm{min}$ down to room temperature. The density of a sample was measured at room temperature by a hydrostatic weighing method. Cyclohexane was used as the immersion liquid, and the density was calibrated by a silicon single crystal with high quality as a standard, therefore the density of a sample could be measured with a high accuracy [11]. The $\mathrm{CsLiB}_{6} \mathrm{O}_{10}$ (CLBO) crystal plates, which is equivalent to $12.5 \mathrm{Li}_{2} \mathrm{O} \cdot 12.5 \mathrm{Cs}_{2} \mathrm{O} \cdot 75 \mathrm{~B}_{2} \mathrm{O}_{3}$, were purchased from Kougakugiken Corp. and two surfaces were optically polished.

The experimental setup of a Brillouin scattering apparatus for high temperatures is shown in Fig. 1. The features of this system are the combination of an optical microscope (Olympus BH-2) and a Sandercock-type $3+3$ passes tandem multipass Fabry-Perot interferometer (FPI). Brillouin scattering was excited by a single frequency green YAG laser with a wavelength of $532 \mathrm{~nm}$ and a power of $50 \mathrm{~mW}$. The spectra were measured at a backward scattering geometry, and this scattering geometry enables the measurement of the longitudinal acoustic (LA) mode. A free spectral range of $60 \mathrm{GHz}$ was applied for measurements, and the scanning range was between -48.8 and $48.8 \mathrm{GHz}$ to acquire a Brillouin spectrum. A conventional photon counting system and a multichannel analyzer were used to detect and accumulate the output signals [12]. To measure spectra at high temperatures, we prepared a compact IR image furnace (Yonekura, IR-TP) specially customized for the light scattering experiments [9].

3. Mixed alkali effect on elastic properties at room temperature

Figure 2 shows the composition dependence of Brillouin scattering spectra of $0.25\left[x \mathrm{Li}_{2} \mathrm{O}-(1-x)\right.$ $\left.\mathrm{Cs}_{2} \mathrm{O}\right]-0.75 \mathrm{~B}_{2} \mathrm{O}_{3}$ glass $(x=0,0.25,0.5,0.75,1)$ at room temperature. The observed spectrum shows a doublet of longitudinal acoustic (LA) mode. The Brillouin scattering spectrum was analyzed by fitting using a Voigt function. The LA velocity $\left(V_{L}\right)$ and its absorption coefficient $\left(\alpha_{\mathrm{L}}\right)$ were calculated from the Brillouin $\operatorname{shift}\left(\Delta v_{180}\right)$ at a backward scattering geometry and the full width at half maximum ( $\Gamma$, FWHM) of a Brillouin peak using the following equations: 


$$
\begin{gathered}
V_{L}=\frac{\Delta v_{180} \lambda}{2 n \sin (\theta / 2)}, \\
\alpha_{L}=\frac{\pi \Gamma}{V_{L}},
\end{gathered}
$$

where $\lambda$ is the wavelength of the incident beam $(532 \mathrm{~nm}), \theta$ is the scattering angle $\left(180^{\circ}\right.$ for backward scattering), and $n$ is the refractive index at $532 \mathrm{~nm}$. According to the paper by Sharaf et al. the value of $n$ for mixed alkali borate glasses shows a linear change with the composition $x$ [14]. Therefore, we used the values of $n$ for single alkali borate glasses reported by Lorosch et al. [13]. The longitudinal modulus $(L)$ was calculated by the equation,

$$
L=\rho \cdot V_{L}^{2}
$$

where $\rho$ is the density of a sample.

Figure 3 shows the composition dependence of observed $\rho$ and $L$ at room temperature. With the increase of $x$, the density shows approximately monotonic decrease. While, $L$ shows the negative deviation from linearity. Such a negative deviation from linearity indicates the presence of MAE, and this fact is in agreement with the previous experimental result of mixed lithium caesium glasses with $x=0.28$ [7]. The values of density $\rho$ and longitudinal modulus $L$ at $x=0$ and 1 are in good agreement with the observed values by Kodama et al.[11,15]. The value of an annealed sample is slightly larger than that of quenched one in all samples, and we used the annealed values for the discussion on MAE.

In the composition dependence of $L$, it is important to discuss the changes of structural units in the borate network [16]. Pure $\mathrm{B}_{2} \mathrm{O}_{3}$ glass is essentially constructed by a planar $\mathrm{BO}_{3}$. The addition of alkali metal oxide causes the formation of $\mathrm{BO}_{4}$ and non-bridging oxygen (NBO). The fraction of $\mathrm{BO}_{4}$ increases monotonically with the increase of alkali composition up to $y=0.3$. In addition, this fraction at the same alkali composition decreases as the size of alkali ion increases. The $\mathrm{BO}_{4}$ units enhance the rigidity of alkali borate glass, because it forms three-dimensional covalent bonds in glass network. Hence, longitudinal modulus $L$ increases with the increase of the alkali composition up to $y=0.3$. The value of $L$ at the same alkali composition decreases as the size of alkali ion increases, because the fraction of $\mathrm{BO}_{4}$ decreases. Consequently, the observed value of $L$ at $x=1.0$ (lithium borate glass) is much larger than that of $L$ at $x=0$ (caesium borate glass).

Another factor for the change of $L$ is the species of alkali ions which control the creation of NBO. We discuss the origin of the deviation from the linearity of $L$ on mixed alkali borate glasses in terms of $\mathrm{BO}_{4}$ and NBO. In the present study, the sum of two alkali compositions is fixed at $y=0.25$. In this composition range, 
the influence of the creation of $\mathrm{NBO}$ on $L$ is much smaller than that of $\mathrm{BO}_{4}$. The fraction of $\mathrm{BO}_{4}$ on mixed alkali borate glasses was determined by Zhong and Bray using NMR [17]. Its fraction of mixed alkali glasses was smaller than the value expected from the value of a single alkali borate glass. The molecular dynamical simulations by Vegiri et al. reported that the abundance of $\mathrm{BO}_{4}$ units decreases from $\mathrm{Li}$ to $\mathrm{Cs}$ and exhibits negative deviation from linearity in Li-Cs glasses [18]. Thus, the negative deviation of $L$ in the present Li-Cs glasses can be essentially attributed to the decrease of the fraction of $\mathrm{BO}_{4}$ caused by the dissimilar alkalis mixing.

In contrast to the negative deviation of $L$, the density shows the linearity approximately as shown in Fig. 3. According to the study of the packing in alkali modified oxide glasses by Giri et al., ionic and covalent packing were discussed in terms of relative volumes of modified cations compared to that of the networking forming oxygen [19]. For modifying ions having volume larger than oxygen $(\mathrm{K}, \mathrm{Rb}$, and $\mathrm{Cs})$, the packing is dominated by modifier alkali ions, and it is called as ionic packing. Therefore, the packing of caesium borate glass, the density is essentially determined by the Cs composition. While, for alkali ions smaller than oxygen ( $\mathrm{Li}$ and $\mathrm{Na}$ ) the packing is controlled by the oxygen covalent network, and it is called as covalent packing. For lithium borate glass the density is essentially determined by not Li composition but the boron-oxygen covalent network. For lithium caesium glass, the situation is more complicated by the coexistence of the smallest and the largest alkali ions. As the composition of Cs increases, the role of covalent packing by Li ions decreases while that of ionic packing by Cs ions increases. Consequently, the approximate linearity of density may hold by the delicate balance between ionic packing and covalent packing without the role of coexistence for packing.

\section{Annealing and temperature variation of longitudinal sound velocity}

The temperature dependence of the Brillouin spectra was measured in a large temperature range between 300 and $1300 \mathrm{~K}$. The typical Brillouin scattering spectra of $0.125 \mathrm{Li}_{2} \mathrm{O}-0.125 \mathrm{Cs}_{2} \mathrm{O}-0.75 \mathrm{~B}_{2} \mathrm{O}_{3}(x=0.5)$ glass, crystal and melt are shown in Fig. 4. The observed spectra of glass and melt include the doublet of longitudinal acoustic (LA) peak, in addition, a central peak (CP) at zero frequency shift was also observed in melt above $1100 \mathrm{~K}$. The peak frequency of LA mode decreases on heating, while its width increases on heating. The intensity of $\mathrm{CP}$ also increases on heating. While, in the spectrum of a crystal shows TA and LA peaks at about 28 and $35 \mathrm{GHz}$, respectively. In the analysis of the temperature dependence of sound velocity and absorption coefficient, since the change of $n$ is much smaller than the change of Brillouin shift, we used the value of $n$ at room temperature for all temperatures.

The difference between quenched and annealed glasses was studied. The annealed glass was prepared by the following process. At first quenched glass was heated from room temperature up to the glass transition temperature $T_{\mathrm{g}}$ with heating rate $1 \mathrm{~K} / \mathrm{min}$, then it was annealed of one hour at $T_{\mathrm{g}}$, then it was cooled down to room temperature with cooling rate $1 \mathrm{~K} / \mathrm{min}$. It is found that after the annealing both LA velocity and density increase slightly at room temperature. However, there is no noticeable change in Raman scattering spectra 
measured before and after annealing. Therefore, no drastic change occurs in short-range structural units and only slight distortion of structural units may be released. However, the remarkable change was observed in the temperature dependence of $V_{\mathrm{L}}$ of $0.125 \mathrm{Li}_{2} \mathrm{O}-0.125 \mathrm{Cs}_{2} \mathrm{O}-0.75 \mathrm{~B}_{2} \mathrm{O}_{3}$ below $800 \mathrm{~K}$ as shown in Fig. $5(\mathrm{a})$. The open and closed triangles indicate the values of $V_{\mathrm{L}}$ of annealed and quenched glasses, respectively. The value of $V_{\mathrm{L}}$ of annealed one monotonically decreases as the temperature increases. The inflection point, at which the rate of decrease of $V_{\mathrm{L}}$ changes drastically changes, is identified as $T_{\mathrm{g}}$. While, the complicated temperature variation was observed for $V_{\mathrm{L}}$ of quenched one. At first, on heating it monotonically decreases below $500 \mathrm{~K}$. Then it turned to increase and approaches the value of annealed one probably owing to gradual annealing near $T_{\mathrm{g}}$. For further heating, it became equal to the value of annealed glass at $T_{\mathrm{g}}$. Above $T_{\mathrm{g}}$, these values change in the same manner reflecting the fact quenched memory disappear completely in a supercooled liquid state. Such an aging behavior was also observed in alkali germanate glasses [10].

Next, we discuss the high temperature behaviors of liquid and crystalline states. Figures 6 shows the temperature dependences of $V_{\mathrm{L}}$ and $\alpha_{\mathrm{L}}$ in $0.25\left[x \mathrm{Li}_{2} \mathrm{O}-(1-x) \mathrm{Cs}_{2} \mathrm{O}\right]-0.75 \mathrm{~B}_{2} \mathrm{O}_{3}$, respectively. The some temperature region above $700 \mathrm{~K}$, the disappearance of Brillouin peaks occurs by the devitrification owing to crystallization. Such devitrification was also reported for pure $\mathrm{B}_{2} \mathrm{O}_{3}$ glass, single alkali borate glasses and other oxide glasses [13]. The characteristic inflection point of sound velocity, which is identified as $T_{\mathrm{g}}$, was observed for all glasses to be studied. In a similar way, the small $\alpha_{\mathrm{L}}$ below $T_{\mathrm{g}}$ increases markedly above $T_{\mathrm{g}}$. The slope of decrease of $V_{\mathrm{L}}$ just above $T_{\mathrm{g}}$ is a direct consequence of structural degradation in a supercooled liquid state. Thus, it could be used for quantitative evaluation of "Fragility" given by Angell [20, 21]. Such a drastic change suggests that the rigidity of the glass network structure becomes weak suddenly above $T_{\mathrm{g}}$, caused by the looseness of the glass network structure. The conversion from $\mathrm{BO}_{4}$ to $\mathrm{BO}_{3}$ and the formation of NBO were observed by the neutron diffraction and Raman scattering studies, thus the breakdown in the glass network above $T_{\mathrm{g}}$ is caused by the creation of random connections and NBO. The $\alpha_{\mathrm{L}}$ gradually increases from $T_{\mathrm{g}}$ to $1300 \mathrm{~K}$. At high temperatures, the $\alpha_{\mathrm{L}}$ of the mixed alkali borate glasses is larger than the value expected from the value of single alkali borate glasses. It is known that the ionic conductivity increases markedly, because alkali ions move freely in a high temperature liquid state. Moreover, MAE enhances the ionic conductivity and it causes the marked increase of attenuation of high frequency LA mode in the GHz range. The increase of $\mathrm{CP}$ intensity can be also attributed the enhaced ionic conductivity at high temperatures. Such enhancement of CP intensity by ionic conduction was reported [22]. In nonlinear optic $\mathrm{KTiOPO}_{4}$ crystals, an intense quasi-elastic light scattering signal, related to the ionic conductivity, was observed in the Brillouin scattering spectra [22]. In a superionic $\mathrm{RbNbWO}_{6}$ crystal with a defect pyrochlore exhibits also the strong central peak comes from $\mathrm{Rb}^{+}$ion hopping [23].

The difference of LA velocity between a melt-quenched glass and a commercially available CLBO crystal was shown in Fig.5 (b) as a function of temperature. The velocity of a CLBO crystal is almost tenperature indelendent below the melting temperature $T_{\mathrm{m}}$ with no anomaly at $T_{\mathrm{g}}$, and at it shows discontinuous drop into an equilibrium liquid phase. It is the typical behavior of the first order phase 
transition from solid to liquid phases.

The temperature dependence of $V_{\mathrm{L}}$ shown in Fig. 6 was scaled in such a way that $V_{\mathrm{L}}$ and $T$ are scaled by $V_{\mathrm{L}}$ at $T_{\mathrm{g}}$ and $T_{\mathrm{g}}$, respectively. Figure 7 shows the scaled temperature dependence of $V_{\mathrm{L}}$. For the melting temperature $T_{\mathrm{m}}$ and $T_{\mathrm{g}}$, the simple empirical relation $T_{\mathrm{m}} / T_{\mathrm{g}} \approx 2 / 3$ holds in general [21]. The scaled temperature dependences of $V_{\mathrm{L}}$ were plotted clearly on the master curve below $T_{\mathrm{g}}$. This result indicates that below $T_{\mathrm{g}}$, the change of $V_{\mathrm{L}}$ is essentially caused not by alkali ions but by the boron-oxide network structure. While above $T_{\mathrm{g}}$, the role of alkali ions is enhanced.

\section{Mixed alkali effect on fragility}

The glass forming materials are classified by fragility on the basis of the temperature dependence of viscosity or primary relaxation time just above $T_{\mathrm{g}}$ according to the concept of "strong" and "fragile" liquids. The temperature dependence of relaxation time of strong liquid obeys the Arrhenius behavior and there is no anomaly in the vicinity of $T_{\mathrm{g}}$. In contrast, the temperature dependence of relaxation time of fragile liquid obeys the Vogel-Fulcher behavior, and the divergence of relaxation time is expected at Vogel-Fulcher temperature or freezing temperature, which is an ideal dynamic glass transition temperature. Since Brillouin scattering measurement can determine longitudinal sound velocity which depends on free volume, the rate of decrease of $V_{\mathrm{L}}$ of a supercooled liquid state just above $T_{\mathrm{g}}$ is a direct consequence of structural degradation in a supercooled liquid. Therefore, the slope of $V_{\mathrm{L}}$ just above $T_{\mathrm{g}}\left(d V_{\mathrm{L}} / d T\right)$ can be used as a measure of fragility. The correlation between the values of $d V_{\mathrm{L}} / d T$ just above $T_{\mathrm{g}}$ and fragility was reported in alkali borate and alkali germinate glasses $[9,10]$.

Figure 8 show the composition dependence of $d V_{\mathrm{L}} / d T$ just above $T_{\mathrm{g}}$. It is found that the composition dependence of $d V_{\mathrm{L}} / d T$ in mixed alkali borate glasses exhibited the deviation from linearity. In addition, the values of $x=0,1$ were in agreement with the values of ref. 9. The fragility of alkali borates glass increases with increasing alkali composition, namely it changes from strong to fragile. In lithium and sodium borate glasses it was reported that the origin of fragility strongly correlates to the formation of the $\mathrm{BO}_{4}[24,25]$. Similar result was also reported in alkali borate binary glasses [9]. Therefore, the deficiency of $\mathrm{BO}_{4}$ units by MAE exhibits the negative deviation from linearity in caesium lithium borate glasses. The fragility relates also to the Gruneisen parameter, and higher anharmonicity corresponds to higher fragility in lithium borate glasses [26]. The correlation between MAE and anharmonicity, which is related to ionic conductivity, is interesting, while further study is necessary to clarify its mechanism.

\section{Conclusion}

The mixed alkali effect on elastic properties of lithium caesium borate glasses $0.25\left[x \mathrm{Li}_{2} \mathrm{O}-(1-x)\right.$ $\left.\mathrm{Cs}_{2} \mathrm{O}\right]-0.75 \mathrm{~B}_{2} \mathrm{O}_{3}$ was studied by Brillouin scattering. In the composition dependences of longitudinal modulus, the mixed alkali effect were observed at room temperature as the negative deviation from linearity, and their mechanism was discussed on the basis of the decrease of $\mathrm{BO}_{4}$ units and the increase of nonbridging 
oxygens. The differences between glasses, crystals and melts were clarified definitely by the temperature dependence of elastic properties measured in the large temperature range from room temperature to $1500 \mathrm{~K}$. The annealing effect of glasses was observed clearly in the temperature variation of LA sound velocity below $T_{\mathrm{g}}$. The composition dependence of fragility was evaluated by the temperature derivative of LA velocity of a supercooled liquid state just above $T_{\mathrm{g}}$, and the negative deviation was found.

\section{Acknowledgement}

Authors are very thankful to Prof. M. Kodama for fruitful discussion.

\section{References}

[1] J.O. Isard, J. Non-Cryst. Solids 1 (1969) 235-261.

[2] D.E. Day, J. Non-Cryst. Solids 21 (1976) 343-372.

[3] G.N. Greaves, K.L. Ngai, J. Non-Cryst. Solids 172 (1994) 1378-1388.

[4] M. Tomozawa, J. Non-Cryst. Solids 196 (1996) 280-284.

[5] M.D. Ingram, B. Roling, J. Phys.: Condens. Matter 15 (2003) S1595-S1606.

[6] J. Swenson and S. Adams, Phys. Rev. Lett. 90 (2003) 155507-1-4.

[7] M. Kodama and S. Kojima, Phys. Chem. Glasses: Eur. J. Glass Sci. Technol. B, 55 (2014), 1-12.

[8] Y. Mori, I. Kuroda, S. Nakajima, T. Sasaki and S. Nakai, Appl. Phys. Lett. 67 (1995) 1818-1820.

[9] S. Kojima, H. Anwar, J. H. Ko, M. Kodama, S. A. Feller, M. Affatigato, R. C. Nieuwendaal, W.J. Clarida and R. Leelasagar, Phys. Chem. Glasses, 44 (2003) 88-90.

[10] M. Kawashima, Y. Matsuda, and S. Kojima: J. Mol. Struct. 993 (2011) 155-159.

[11] K. Kaneda, K. Arae, M. Kodama, and S. Kojima, J. Non-Crys. Solids. 358 (2012) 799-803.

[11] M. Kodama and S. Kojima, Jpn. J. Appl. Phys. 34 (1995) 2570-2574.

[12] S. Kojima, Jpn. J. Appl. Phys. 49 (2010) 07HA01-1-6.

[13] J. Lorosch, M. Couzi, J. Pelous, R. Vacher, A. Levasseur, J. Non-Cryst. Solids 69 (1984) 1-25.

[14] N. A. Sharaf, A. A. Ahmed and A. F. Abbas, Phys. Chem. Glasses, 39 (1998) 76-82.

[15] M. Kodama, Jpn. J. Appl. Phys. 32 (1993) 2227-2280.

[16] Ed. by Adrian C. Wright, Steven A. Feller, Alex C. Hannon, Proceedings of the Second International Conference on Borate Glasses, Crystals and Melts, Society of Glass Technology, Sheffield, 1997.

[17] J. Zhong and P. J. Bray, J. Non-Cryst. Solids, 111 (1989) 67-76.

[18] A. Vegiri, C-P. E. Varsamis and E. I. Kamitsos, Phys. Rev. B 80 (2009) 184202-1-12.

[19] S. Giri, C. Gaebler, J. Helmus, M. Affatigato, S. Feller, and M. Kodama, J. Non-Crys. Solids 347 (2004) 87-92.

[20] C. A. Angell, J. Non-Cryst. Solids. 131-133 (1991) 13-31.

[21] S. Sakka and J. D. Machenzie, J. Non-Cryst. Solids, 6 (1971) 145-162.

[22] M. Serhane and P. Moch, J. Phys.: Condens. Matter 6 (1994) 3821-3830. 
[23] M. Mạczka, J.-H. Ko, S. Kojima, J. Hanuza, and A. Majchrowski, J. Appl. Phys. 94 (2003) 3781-3784.

[24] Y. Matsuda, Y. Fukawa, M. Kawashima, S. Mamiya and S. Kojima, Solid State Ionics, 179 (2008) 2424-2427.

[25] Y. Fukawa, Y. Matsuda, M. Kawashima, S. Kojima, J. Therm. Anal. Calorim. 99 (2010) 39-44.

[26] S. Kojima, V. N. Novikov, and M. Kodama, J. Chem. Phys. 113 (2000) 6344-6350.

Figure captions

Fig. 1 Schematic diagram of Brillouin scattering setup for high-temperature measurement. (a) Compact IR furnace, and (b) micro-Brillouin scattering setup.

Fig. 2 Composition dependence of Brillouin scattering spectra of $25\left\{x \mathrm{Li}_{2} \mathrm{O} \cdot(1-x) \mathrm{Cs}_{2} \mathrm{O}\right\} \cdot 75 \mathrm{~B}_{2} \mathrm{O}_{3}$ at room temperature.

Fig. 3 Composition dependences of density and longitudinal modulus of $25\left\{\mathrm{xLi}_{2} \mathrm{O} \cdot(1-\mathrm{x}) \mathrm{Cs}_{2} \mathrm{O}\right\} \cdot 75 \mathrm{~B}_{2} \mathrm{O}_{3}$ glasses.

Fig. 4 Brillouin scattering spectra of $12.5 \mathrm{Li}_{2} \mathrm{O} \cdot 12.5 \mathrm{Cs}_{2} \mathrm{O} \cdot 75 \mathrm{~B}_{2} \mathrm{O}_{3}$ glasses, crystals, and melts at various temperature. The Brillouin spectrum of a crystal shows TA and LA peaks at about 28 and $35 \mathrm{GHz}$, respectively.

Fig. 5 Temperature dependence of Brillouin shift of $12.5 \mathrm{Li}_{2} \mathrm{O} \cdot 12.5 \mathrm{Cs}_{2} \mathrm{O} \cdot 75 \mathrm{~B}_{2} \mathrm{O}_{3}$ glasses at various temperatures, (a) before and after annealing, (b) difference between crystal and glass at high temperatures.

Fig. 6 Longitudinal sound velocity and absorption coefficient of $25\left\{x \mathrm{Li}_{2} \mathrm{O} \cdot(1-x) \mathrm{Cs}_{2} \mathrm{O}\right\} \cdot 75 \mathrm{~B}_{2} \mathrm{O}_{3}$ glass as a function of the temperature

Fig. 7 Scaled temperature dependence of $V_{\mathrm{L}}$ of $25\left\{x \mathrm{Li}_{2} \mathrm{O} \cdot(1-x) \mathrm{Cs}_{2} \mathrm{O}\right\} \cdot 75 \mathrm{~B}_{2} \mathrm{O}_{3}$ in such a way that $V_{\mathrm{L}}$ and $T$ are scaled by $V_{\mathrm{L}}$ at $T_{\mathrm{g}}$ and $T_{\mathrm{g}}$, respectively

Fig. 8 The composition variation of $\mathrm{dV}_{\mathrm{L}} / \mathrm{dT}$ just above $T_{\mathrm{g}}$ as a measure of fragility. 


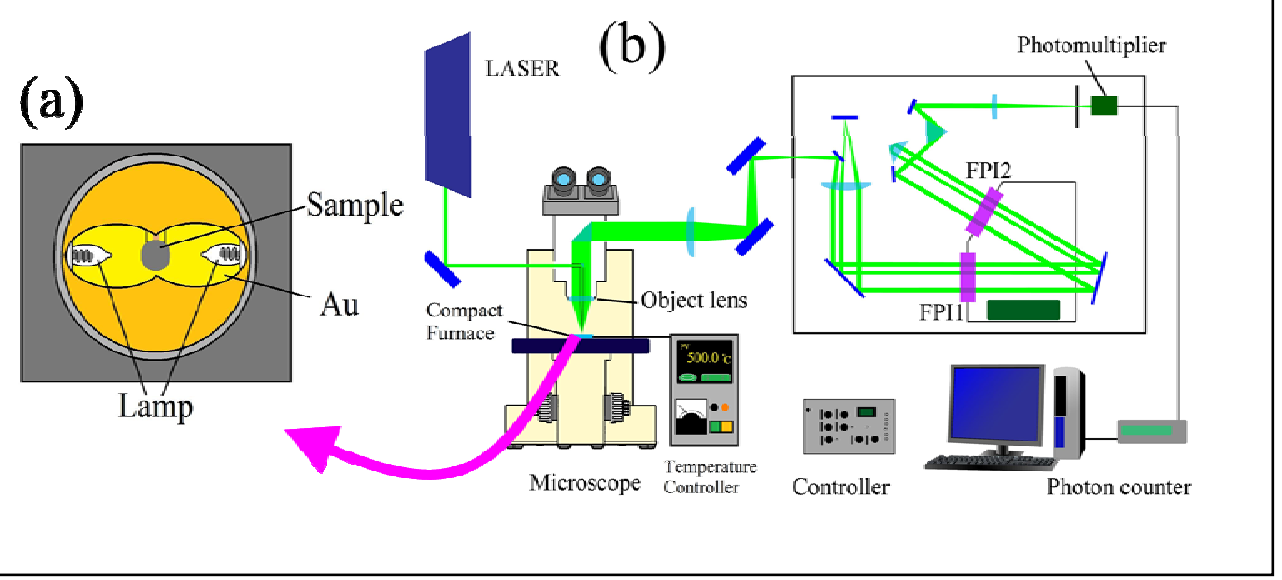

Fig. 1

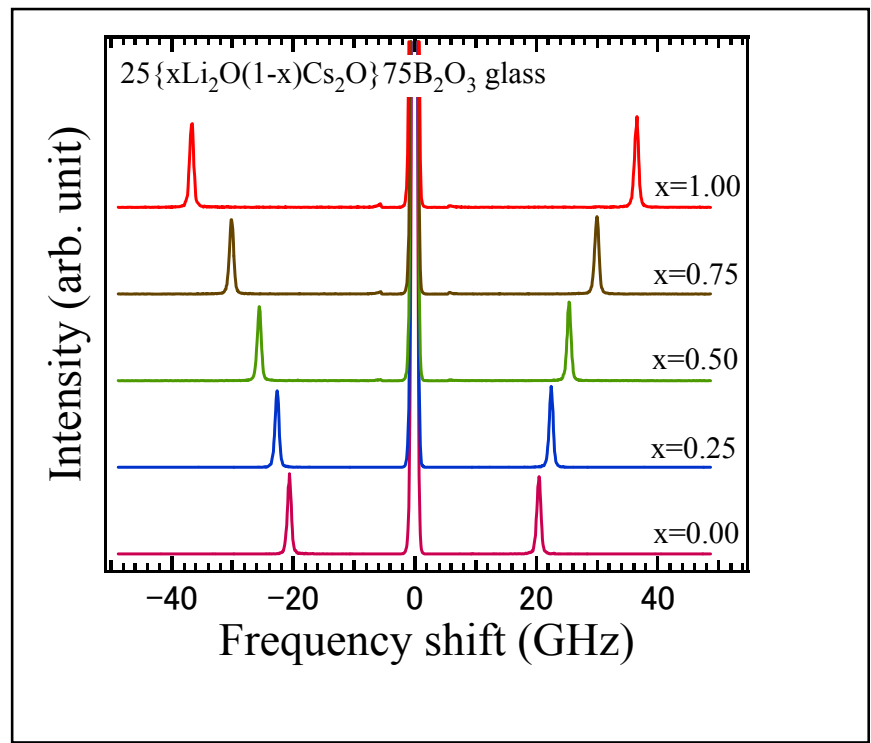

Fig. 2

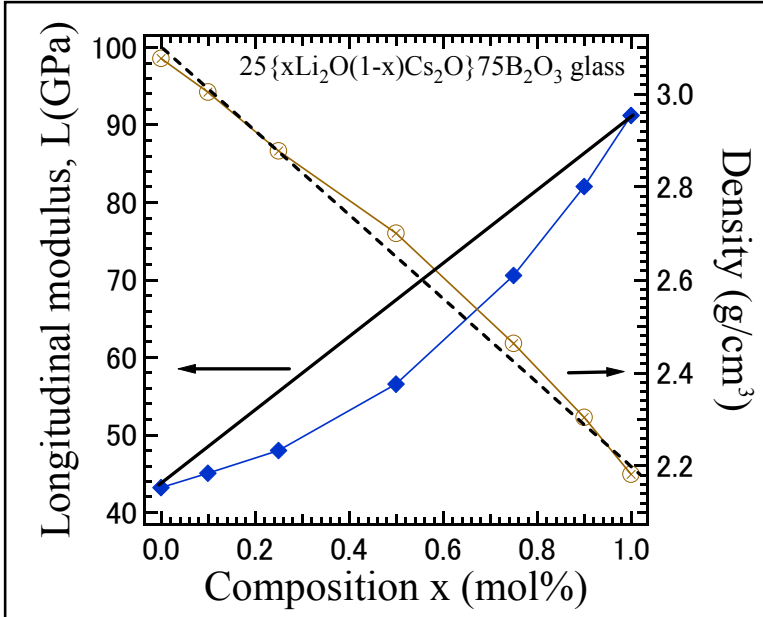

Fig. 3 


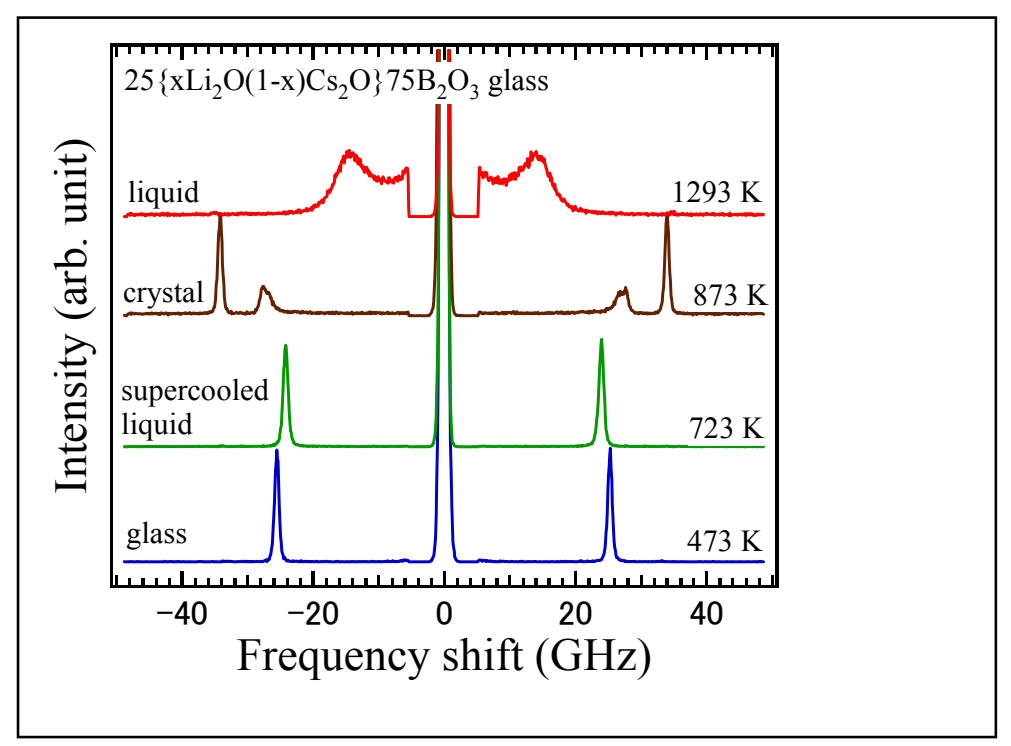

Fig. 4

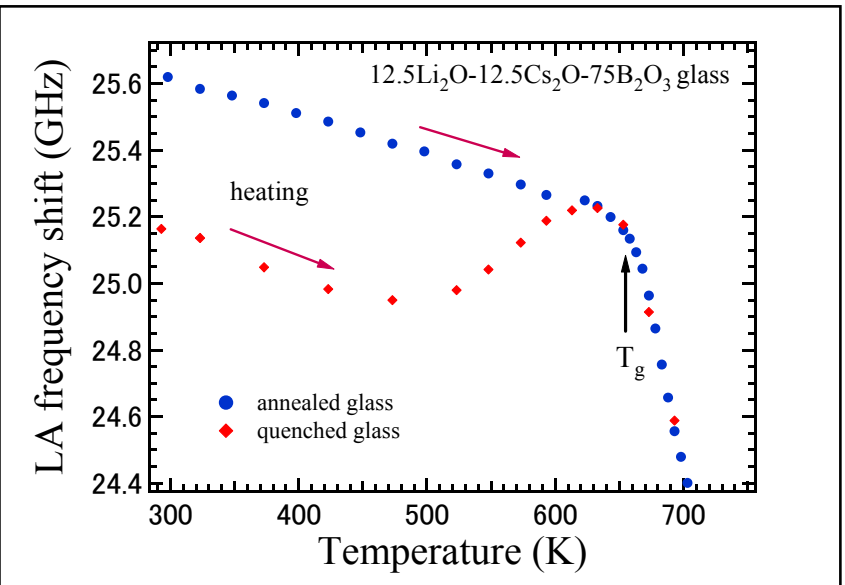

Fig. 5(a)

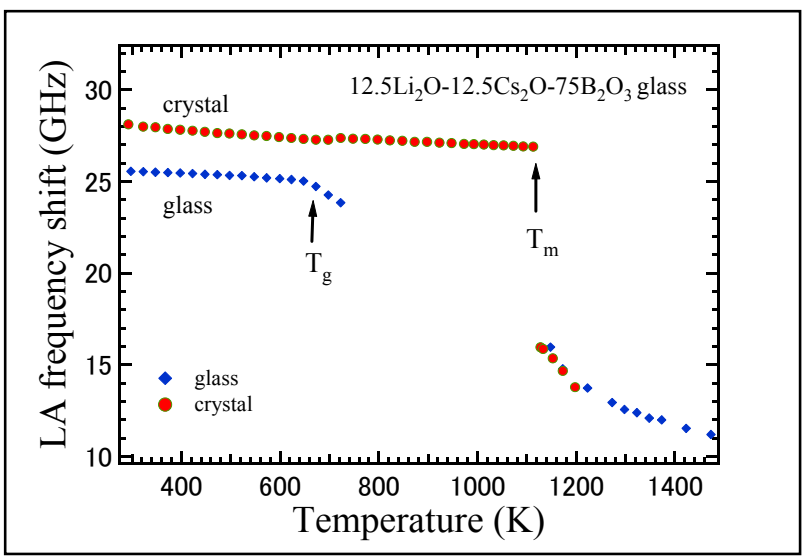

Fig. 5(b) 


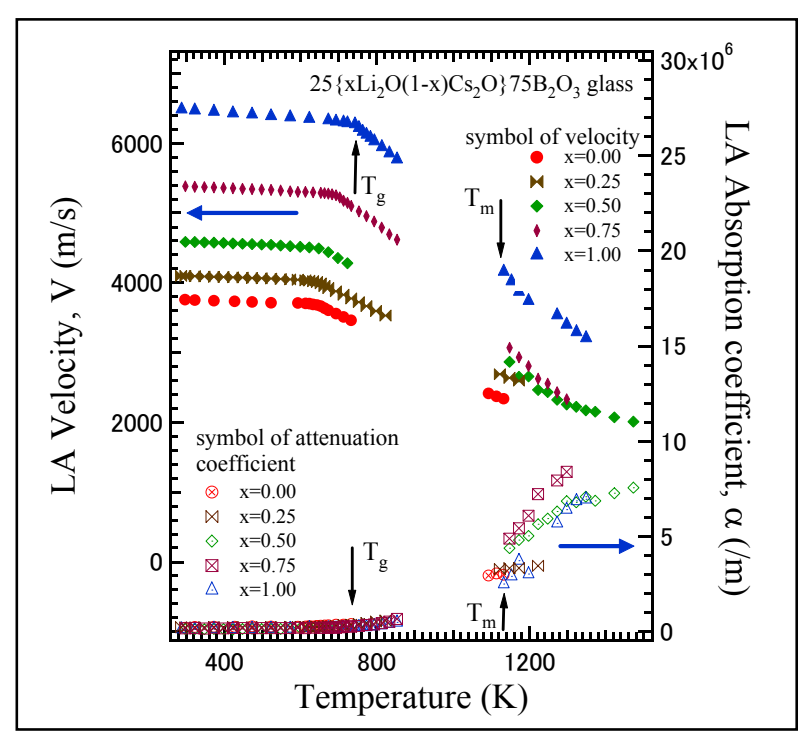

Fig. 6

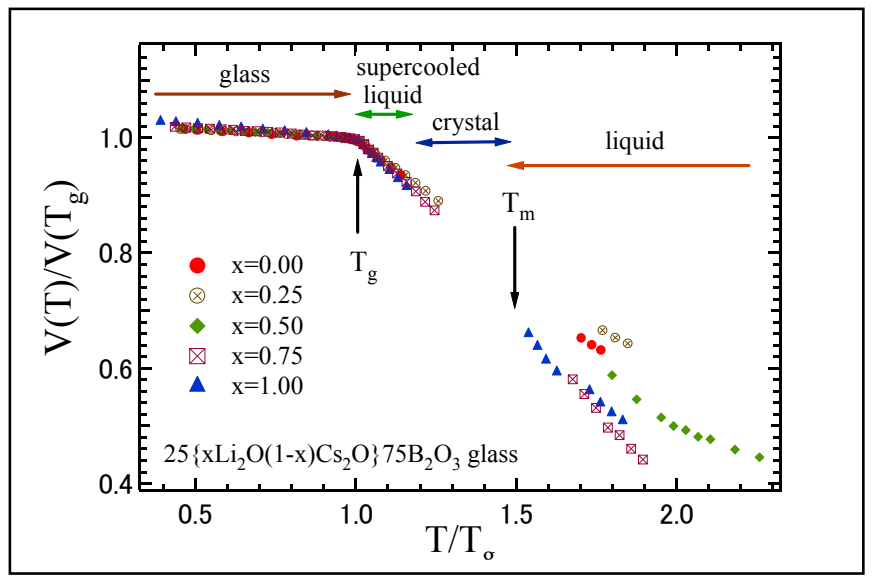

Fig. 7

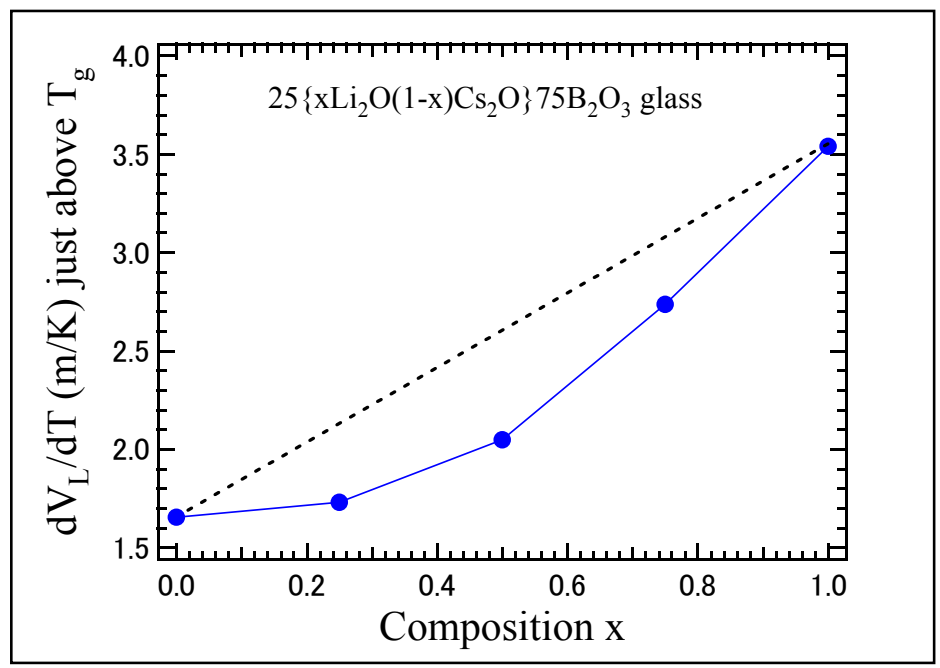

Fig. 8 